\title{
ON SOME GENERALISATIONS OF THE RIEMANN-LIOUVILLE AND WEYL FRACTIONAL INTEGRALS AND THEIR APPLICATIONS
}

\author{
by J. S. LOWNDES
}

(Received 19 January, 1980)

1. For functions $f \in L_{\mathrm{Loc}}[0, \infty)$ the Riemann-Liouville operator of fractional integration $I^{\alpha}$ is defined by

$$
I^{\alpha} f(x)=\frac{2}{\Gamma(\alpha)} \int_{0}^{x}\left(x^{2}-t^{2}\right)^{\alpha-1} t f(t) d t \quad(\alpha>0)
$$

and its adjoint operator, the Weyl operator $K^{\alpha}$, is defined by

$$
K^{\alpha} f(x)=\frac{2}{\Gamma(\alpha)} \int_{x}^{\infty}\left(t^{2}-x^{2}\right)^{\alpha-1} \mathrm{tf}(t) d t \quad(\alpha>0)
$$

for functions $f \in L_{\mathrm{Loc}}(0, \infty)$ having a suitable behaviour at infinity.

These operators and their modifications have been extensively discussed within a framework of generalised functions by Erdélyi and McBride $[3,4,7,8]$. However, for our purposes, it will be sufficient to proceed along the lines adopted by Erdélyi in his earlier papers $[1,2]$ and to consider fractional integrals of functions which are continuous on the open interval $(0, \infty)$ and subject to growth conditions at zero and infinity.

The above operators satisfy the basic relations

$$
\begin{aligned}
I^{0} f & =f, \quad K^{0} f=f, \\
I^{\alpha} I^{\beta} f & =I^{\alpha+\beta} f, \quad K^{\alpha} K^{\beta} f=K^{\alpha+\beta} f
\end{aligned}
$$

and a summary of their other properties is given in [1], where it is shown that they are related to the Erdélyi-Kober operators $I_{n, \alpha}$ and $K_{n, \alpha}$ through the formulae

$$
\begin{gathered}
I_{\eta, \alpha} f=x^{-2(\alpha+\eta)} I^{\alpha} x^{2 \eta} f, \\
K_{\eta, \alpha} f=x^{2 \eta} K^{\alpha} x^{-2(\alpha+\eta)} f .
\end{gathered}
$$

A comprehensive account of the operators $I_{n, \alpha}$ and $K_{n, \alpha}$ and a survey of their applications can be found in the book [14] and review article [15] by Sneddon.

In this paper a discussion of some generalisations of the operators $I^{\alpha}$ and $K^{\alpha}$ is presented together with a brief account of their applications in the solution of partial differential equations.

2. For functions $f \in C(0, \infty)$ and having a suitable behaviour at zero and infinity, we now introduce the generalised Riemann-Liouville operators $I_{\lambda}^{\alpha}$, together with their adjoint operators, the generalised Weyl operators $K_{\lambda}^{\alpha}$, where $\lambda=k$ or $\lambda=i k, k \geqslant 0$. These are

Glasgow Math. J. 22 (1981) 173-180. 
defined by the formulae

$$
\begin{array}{rlr}
I_{\lambda}^{\alpha} f(x) & =2^{\alpha} k^{1-\alpha} \int_{0}^{x} u\left(x^{2}-u^{2}\right)^{(\alpha-1) / 2} G_{\lambda}\left\{k \sqrt{ }\left(x^{2}-u^{2}\right)\right\} f(u) d u & (\alpha>0), \\
& =\mathscr{D}^{m} I_{\lambda}^{\alpha+m} f(x) \quad(\alpha<0), \\
K_{\lambda}^{\alpha} f(x) & =2^{\alpha} k^{1-\alpha} \int_{x}^{\infty} u\left(u^{2}-x^{2}\right)^{(\alpha-1) / 2} G_{\lambda}\left\{k \sqrt{ }\left(u^{2}-x^{2}\right)\right\} f(u) d u & (\alpha>0), \\
& =(-1)^{m} \mathscr{D}^{m} K_{\lambda}^{\alpha+m} f(x) \quad(\alpha<0),
\end{array}
$$

where $G_{k}(z)=J_{\alpha-1}(z)$ is the Bessel function of the first kind, $G_{i k}(z)=I_{\alpha-1}(z)$ is the modified Bessel function of the first kind, $m$ is a positive integer such that $0<\alpha+m<1$ and $\mathscr{D}$ denotes the differential operator $\mathscr{D}=\frac{1}{2 x} \frac{d}{d x}$.

It is easily seen that

$$
I_{0}^{\alpha} f=I^{\alpha} f, \quad K_{0}^{\alpha} f=K^{\alpha} f
$$

and other properties of $I_{\lambda}^{\alpha}$ and $K_{\lambda}^{\alpha}$ can be deduced from the corresponding properties of the generalised Erdélyi-Kober operators $\mathfrak{I}_{\lambda}(\eta, \alpha)$ and $\mathfrak{A}_{\lambda}(\eta, \alpha)$ which are given in [5], since they are related through the equations

$$
\begin{aligned}
& \Im_{\lambda}(\eta, \alpha) f(x)=x^{-2(\alpha+\eta)} I_{\lambda}^{\alpha} x^{2 \eta} f(x), \\
& \mathfrak{I}_{\lambda}(\eta, \alpha) f(x)=x^{2 \eta} K_{\lambda}^{\alpha} x^{-2(\alpha+\eta)} f(x) .
\end{aligned}
$$

3. We now investigate the forms that the operators take when $\alpha=0$ and, in order to do this, we consider the expressions

$$
\begin{gathered}
I_{k}^{\alpha+1} \mathscr{D} f(x)=2^{\alpha} k^{-\alpha} \int_{0}^{x}\left(x^{2}-u^{2}\right)^{\alpha / 2} J_{\alpha}\left\{k \sqrt{ }\left(x^{2}-u^{2}\right)\right\} f^{\prime}(u) d u \\
K_{k}^{\alpha+1} \mathscr{D} f(x)=2^{\alpha} k^{-\alpha} \int_{x}^{\infty}\left(u^{2}-x^{2}\right)^{\alpha / 2} J_{\alpha}\left\{k \sqrt{ }\left(u^{2}-x^{2}\right)\right\} f^{\prime}(u) d u
\end{gathered}
$$

where $\alpha>0$ and $k \geqslant 0$.

On performing an integration by parts on each of the equations, under the assumptions that $f(0)=0$ and $x^{\alpha-1 / 2} f(x) \rightarrow 0$ as $x \rightarrow \infty$, the integrated parts vanish and we are left with the results

$$
I_{k}^{\alpha+1}(\mathscr{D} f)=I_{k}^{\alpha} f, \quad K_{k}^{\alpha+1}(\mathscr{D} f)=-K_{k}^{\alpha} f
$$

Since the left hand sides of these equations are defined for $\alpha>-1$ they can be used to define the operators $I_{k}=I_{k}^{0}$ and $K_{k}=K_{k}^{0}$ to be

$$
\begin{aligned}
I_{k} f(x) & =I_{k}^{1} \mathscr{D} f(x)=\int_{0}^{x} J_{0}\left\{k \sqrt{ }\left(x^{2}-u^{2}\right)\right\} f^{\prime}(u) d u \\
& =f(x)-k \int_{0}^{x} \frac{u}{\left(x^{2}-u^{2}\right)^{1 / 2}} J_{1}\left\{k \sqrt{ }\left(x^{2}-u^{2}\right)\right\} f(u) d u
\end{aligned}
$$


when $f(0)=0$ and

$$
\begin{aligned}
K_{k} f(x) & =-K_{k}^{1} \mathscr{D} f(x)=-\int_{x}^{\infty} J_{0}\left\{k \sqrt{ }\left(u^{2}-x^{2}\right)\right\} f^{\prime}(u) d u \\
& =f(x)-k \int_{x}^{\infty} \frac{u}{\left(u^{2}-x^{2}\right)^{1 / 2}} J_{1}\left\{k \sqrt{ }\left(u^{2}-x^{2}\right)\right\} f(u) d u
\end{aligned}
$$

when $x^{-1 / 2} f(x) \rightarrow 0$ as $x \rightarrow \infty$.

In a similar way we can also define the operators

$$
I_{i k} f(x)=I_{i k}^{1} \mathscr{D} f(x), \quad K_{i k} f(x)=-K_{i k}^{1} \mathscr{D} f(x),
$$

where for the last result we must require that $f(x)=O\left(e^{-\delta x}\right)$ as $x \rightarrow \infty$ where $\delta>k \geqslant 0$.

Vekua [16] has applied an operator of the form

$$
I_{k} f=\left(I_{i k}\right)^{-1} f
$$

in the solution of differential equations and for this reason we can call the associated operator

$$
K_{k} f=\left(K_{i k}\right)^{-1} f
$$

an operator of the Vekua-type.

Two important results connecting the operators $I_{\lambda}^{\alpha}, K_{\lambda}^{\alpha}$ and the singular differential operator

$$
L_{\eta}=x^{-2 \eta-1} \frac{d}{d x} x^{2 \eta+1} \frac{d}{d x}
$$

will now be stated without proof. They are

$$
\begin{aligned}
I_{\lambda}^{\alpha} L_{n} f(x) & =\left(L_{n-\alpha}+\lambda^{2}\right) I_{\lambda}^{\alpha} f(x), \\
K_{\lambda}^{\alpha} L_{n} f(x) & =\left(L_{n-\alpha}-\lambda^{2}\right) K_{\lambda}^{\alpha} f(x),
\end{aligned}
$$

where $\lambda=k$ or $\lambda=i k, k \geqslant 0$.

A relation of the type (12) has been investigated by the author [6] and both expressions are generalisations of results obtained by Erdélyi [1, 2].

For the applications of the operators to be considered later in this paper it will only be necessary to examine special cases of the equations (12) and (13). This is done in the following three theorems and since their proofs follow along similar lines we shall only give a proof of Theorem 2 .

THEOREM 1. If $f \in C^{2}(0, b), b>0$ and $f(0)=f^{\prime \prime}(0)=0$, then

$$
I_{\lambda} f^{\prime \prime}(x)=\left(\frac{d^{2}}{d x^{2}}+\lambda^{2}\right) I_{\lambda} f(x),
$$

where $\lambda=k$ or $\lambda=i k, k \geqslant 0$. 
THEOREM 2. Let $f \in C^{2}(b, \infty), b>0$ and $x^{-1 / 2} f(x) \rightarrow 0, x^{1 / 2} f^{\prime}(x) \rightarrow 0$ and $x^{-1 / 2} f^{\prime \prime}(x) \rightarrow 0$ as $x \rightarrow \infty$; then

$$
K_{k} f^{\prime \prime}(x)=\left(\frac{d^{2}}{d x^{2}}-k^{2}\right) K_{k} f(x) \quad(k \geqslant 0)
$$

Proof. We write

$$
h(x)=K_{k} f(x)=-x \int_{1}^{\infty} J_{0}(k x \xi) f^{\prime}(x t) d t
$$

where $\xi=\sqrt{ }\left(t^{2}-1\right)$.

Since $h(x)$ is differentiable we have

$$
\begin{aligned}
h^{\prime}(x)= & \int_{1}^{\infty}\left[k x \xi J_{1}(k x \xi)-J_{0}(k x \xi)\right] f^{\prime}(x t) d t \\
& -x \int_{1}^{\infty} t J_{0}(k x \xi) f^{\prime \prime}(x t) d t
\end{aligned}
$$

Evaluating the last term by integration by parts we find that the above equation becomes

$$
h^{\prime}(x)=f^{\prime}(x)-x k \int_{1}^{\infty} \xi^{-1} J_{1}(k x \xi) f^{\prime}(x t) d t .
$$

A further differentiation yields the result

$$
\begin{aligned}
h^{\prime \prime}(x) & =f^{\prime \prime}(x)-x k \int_{1}^{\infty} t \xi^{-1} J_{1}(k x \xi) f^{\prime \prime}(x t) d t-x k^{2} \int_{1}^{\infty} J_{0}(k x \xi) f^{\prime}(x t) d t \\
& =K_{k}\left[f^{\prime \prime}(x)+k^{2} f(x)\right]
\end{aligned}
$$

by the definition (8) and this proves the theorem.

THEOREM 3. Let $f \in C^{2}(b, \infty), b>0$ and $f^{(n)}(x)=O\left(e^{-\delta x}\right)$ as $x \rightarrow \infty$, where $n=0,1,2$ and $\delta>k \geqslant 0$; then

$$
K_{i k} f^{\prime \prime}(x)=\left(\frac{d^{2}}{d x^{2}}+k^{2}\right) K_{i k} f(x)
$$

Similar results to those contained in Theorem 1 have been used by Vekua [16] and in a more general sense by the author [6] in the solution of differential equations and so from now on we shall confine our attention to a description of some applications of Theorems 2 and 3 . If we denote the Laplacian operator in $\mathbf{R}^{\mathbf{n}}$ by

$$
\Delta_{n}=\frac{\partial^{2}}{\partial x^{2}}+\sum_{i=1}^{n-1} \frac{\partial^{2}}{\partial x_{i}^{2}}
$$

we can, on using Theorems 2 and 3 , deduce that if $u(r)$ is a solution of the $n$-dimensional 
Laplace equation

$$
\Delta_{n} u(r)=0,
$$

then the corresponding solution of the $n$-dimensional "Helmholtz" equation

$$
\left(\Delta_{n}-\lambda^{2}\right) v(r)=0 \text {, }
$$

is given by $v(r)=K_{\lambda} u(r)$, under appropriate conditions on the function $u(r)$.

4. First of all we give two applications of Theorem 2 .

(i) The function $u(x, y)=-\frac{1}{2} \log \left(x^{2}+y^{2}\right)$ is a fundamental solution of the equation

$$
\Delta_{2} u(x, y)=\left(\frac{\partial^{2}}{\partial x^{2}}+\frac{\partial^{2}}{\partial y^{2}}\right) u=0
$$

which satisfies the conditions of Theorem 2 . We find that

$$
\begin{aligned}
v(x, y) & =K_{k} u(x, y)=\frac{1}{2} \int_{x}^{\infty} J_{0}\left\{k \sqrt{ }\left(t^{2}-x^{2}\right)\right\} \frac{\partial}{\partial t}\left[\log \left(t^{2}+y^{2}\right)\right] d t \\
& =\int_{0}^{\infty} \frac{\xi J_{0}(k \xi)}{\xi^{2}+x^{2}+y^{2}} d \xi=K_{0}\left\{k \sqrt{ }\left(x^{2}+y^{2}\right)\right\},
\end{aligned}
$$

where $K_{0}(z)$ is the modified Bessel function of the second kind, is a fundamental solution of the equation

$$
\left(\Delta_{2}-k^{2}\right) v(x, y)=0 \quad(k \geqslant 0) .
$$

(ii) Similarly, since $u(x, y, z)=r^{-1}=\left(x^{2}+y^{2}+z^{2}\right)^{-1 / 2}$ is a fundamental solution of the equation

$$
\Delta_{3} u(x, y, z)=\left(\frac{\partial^{2}}{\partial x^{2}}+\frac{\partial^{2}}{\partial y^{2}}+\frac{\partial^{2}}{\partial z^{2}}\right) u=0
$$

it follows that

$$
v(x, y, z)=K_{k} u(x, y, z)=\int_{0}^{\infty} \frac{\xi J_{0}(k \xi)}{\left(\xi^{2}+r^{2}\right)^{3 / 2}} d \xi=\frac{e^{-k r}}{r}
$$

is a fundamental solution of the equation

$$
\left(\Delta_{3}-k^{2}\right) v(x, y, z)=0 \quad(k \geqslant 0) .
$$

5. In a number of papers Meister $[10,11,12,13]$ has considered problems concerning subsonic flow past aerofoils arranged in cascades or in a wind tunnel, all of which he reduced to mixed boundary value problems involving the Helmholtz equation. Using integral transforms and the Wiener-Hopf technique he showed that the solutions of these problems can be expressed in terms of the Green's functions of the corresponding boundary value problems for the Laplace equation. 
In what follows we shall discuss two simple boundary value problems involving Laplace's equation which are elementary analogues of Meister's problems. From the solutions of these problems it will be shown, by using the result of Theorem 3 , that the solutions of the corresponding boundary value problems for the Helmholtz equation can easily be obtained.

(i) First of all we examine Laplace's equation in a semi-infinite strip

$$
\left(\frac{\partial^{2}}{\partial x^{2}}+\frac{\partial^{2}}{\partial y^{2}}\right) \phi(x, y)=0 \quad(0<x<\infty, 0<y<1)
$$

with the boundary conditions

$$
\begin{gathered}
\phi(0, y)=\phi_{x}(0, y)=0 \quad(0<y<1), \\
\phi_{y}(x, 0)=\phi_{y}(x, 1) \quad(0<x<\infty), \\
\phi(x, 0)-\phi(x, 1)=2 f(x) \quad(0<x<\infty),
\end{gathered}
$$

where $f(x)$ is a known function. Applying the Laplace transform in $x$

$$
\Phi(s, y)=\int_{0}^{\infty} \phi(x, y) e^{-s x} d x=L[\phi(x, y): x \rightarrow s],
$$

to equation (17) and using the conditions (18) to (20), it can easily be shown that

where $F(s)=L[f(x): x \rightarrow s]$.

$$
\Phi(s, y)=F(s) \frac{\sin \left[s\left(\frac{1}{2}-y\right)\right]}{\sin s / 2},
$$

Inverting the above equation with respect to the Laplace transform and using the convolution theorem we can write the solution of the problem in the form

$$
\phi(x, y)=\int_{0}^{x} f(t) G(x-t, y) d t \quad(0<x<\infty, 0<y<1),
$$

where $G(x, y)$ is the Green's function of the problem given by

$$
\begin{aligned}
G(x, y) & =L^{-1}\left[\frac{\sin \left[s\left(\frac{1}{2}-y\right)\right]}{\sin s / 2}: s \rightarrow x\right] \\
& =2 \sum_{n=1}^{\infty} \sin (2 \pi n y) e^{-2 \pi n x} \\
& =\frac{\sin (2 \pi y)}{\cosh (2 \pi x)-\cos (2 \pi y)} .
\end{aligned}
$$

Now $G(x, y)=O\left(e^{-2 \pi x}\right)$ as $x \rightarrow \infty$ and, since the series (22) is absolutely and uniformly convergent when $x>0$, we can apply Theorem 3 to obtain

$$
\begin{aligned}
K_{i k} G(x, y) & =G_{k}(x, y)=-\int_{x}^{\infty} I_{0}\left\{k \sqrt{ }\left(u^{2}-x^{2}\right)\right\} \frac{\partial}{\partial u} G(u, y) d u \\
& =4 \pi \sum_{n=1}^{\infty} n \sin (2 \pi n y) \int_{x}^{\infty} I_{0}\left\{k \sqrt{ }\left(u^{2}-x^{2}\right)\right\} e^{-2 \pi n u} d u .
\end{aligned}
$$


Evaluating the integral, by using a result given in [9], we find that when $0 \leqslant k<2 \pi$

$$
G_{k}(x, y)=4 \pi \sum_{n=1}^{\infty} \frac{n \sin (2 \pi n y)}{N} e^{-N x},
$$

where $N=\sqrt{ }\left[(2 \pi n)^{2}-k^{2}\right]$.

It is easily verified that the function

$$
\psi(x, y)=\int_{0}^{x} f(t) G_{k}(x-t, y) d t
$$

is the corresponding solution of the Helmholtz equation

$$
\left(\frac{\partial^{2}}{\partial x^{2}}+\frac{\partial^{2}}{\partial y^{2}}+k^{2}\right) \psi(x, y)=0 \quad(0<x<\infty, 0<y<1)
$$

when $0 \leqslant k<2 \pi$ and $\psi(x, y)$ satisfies the boundary conditions (18) to (20).

(ii) As a final example we consider the solution of Laplace's equation (17) with the boundary conditions (18), (19) and

$$
\phi_{y}(x, 0)=g(x) \quad(0<x<\infty),
$$

where $g(x)$ is a prescribed function.

Using the Laplace transform it can be shown that the solution to this problem is

$$
\phi(x, y)=\int_{0}^{x} g(t) H(x-t, y) d t \quad(0<x<\infty, 0<y<1),
$$

where $H(x, y)$ is the Green's function given by

$$
\begin{aligned}
H(x, y) & =L^{-1}\left[\frac{\sin \left[s\left(y-\frac{1}{2}\right)\right]}{s \cos s / 2}: s \rightarrow x\right] \\
& =-\frac{2}{\pi} \sum_{m=1}^{\infty} \frac{\cos [(2 m-1) \pi y]}{(2 m-1)} e^{-(2 m-1) \pi x} \\
& =\frac{1}{2 \pi} \log \left[\frac{\cosh (\pi x)-\cos (\pi y)}{\cosh (\pi x)+\cos (\pi y)}\right] .
\end{aligned}
$$

Since $H(x, y)=O\left(e^{-\pi x}\right)$ as $x \rightarrow \infty$ and the series (25) is absolutely and uniformly convergent when $x>0$, we can use Theorem 3 to find that

$$
\begin{aligned}
K_{i k} H(x, y) & =-2 \sum_{m=1}^{\infty} \cos [(2 m-1) \pi y] \int_{x}^{\infty} I_{0}\left\{k \sqrt{ }\left(u^{2}-x^{2}\right)\right\} e^{-(2 m-1) \pi u} d u \\
& =-2 \sum_{m=1}^{\infty} \frac{\cos [(2 m-1) \pi y]}{M} e^{-M x} \\
& =H_{k}(x, y) \quad(0 \leqslant k<\pi),
\end{aligned}
$$

where $M=\sqrt{ }\left[(2 m-1)^{2} \pi^{2}-k^{2}\right]$. 
In this way we see that when $0 \leqslant k<\pi$ the solution of the Helmholtz equation (23) with the boundary conditions (18), (19) and (24) is given by

$$
\psi(x, y)=\int_{0}^{x} g(t) H_{k}(x-t, y) d t \quad(0<x<\infty, 0<y<1),
$$

where $H_{k}(x, y)$ is defined by equation (26).

\section{REFERENCES}

1. A. Erdélyi, Some applications of fractional integrals, Mathematical Note No. 316, (Boeing Scientific Research Laboratories, 1963).

2. A. Erdélyi, An application of fractional integrals, J. Analyse Math. 14 (1965), 113-126.

3. A. Erdélyi and A. C. McBride, Fractional integrals of distributions, SIAM J. Math. Anal. 1 (1970), 547-557.

4. A. Erdélyi, Fractional integrals of generalized functions, in Lecture Notes in Mathematics No. 457 (Springer-Verlag, 1975), 151-170.

5. J. S. Lowndes, A generalisation of the Erdélyi-Kober operators, Proc. Edinburgh Math. Soc. 17 (1970), 139-148. $35-41$.

6. J. S. Lowndes, An application of some fractional integrals, Glasgow Math. J. 20 (1979),

7. A. C. McBride, A theory of fractional integration for generalised functions, SIAM J. Math. Anal. 6 (1975), 583-599.

8. A. C. McBride, A theory of fractional integration for generalised functions, II, Proc. Roy. Soc. Edinburgh Sect. A 77 (1977), 335-349.

9. W. Magnus, F. Oberhettinger and R. P. Soni, Formulas and theorems for the special functions of mathematical physics, 3rd ed. (Springer-Verlag, 1966).

10. E. Meister, Beitrag zur Aerodynamik eines schwingenden, Gitters II (Unterschallströmung), Z. Angew Math. Mech. 42 (1962), 9-31.

11. E. Meister, Beitrag zur Aerodynamik eines schwingenden, Gitters III (Unterschallströmung), Z. Angew Math. Mech. 42 (1962), 245-254.

12. E. Meister, Zur Theorie der ebenen instationären Unterschallströmung um ein schwingendes Profil im Kanal. Z. Angew Math. Phys. 16 (1965), 770-780.

13. E. Meister, Neuere Ergebnisse der mathematischen Theorie instationärer Gitterströmungen, Acta Mech. 3 (1967), 325-341.

14. I. N. Sneddon, Mixed boundary value problems in potential theory (North Holland, 1966).

15. I. N. Sneddon, The use in mathematical physics of Erdélyi-Kober operators and some of their generalizations, in Lecture notes in mathematics No. 457 (Springer-Verlag, 1975), 37-79.

16. I. Vekua, New Methods for solving elliptic equations, (North Holland 1967).

DePARTMENT OF MATHEMATICS

UNIVERSTYY OF STRATHCLYDE

GLASGOW. 